\title{
Retos y cambios en la organización universitaria. Hacia un nuevo modelo de enseñanza-aprendizaje
}

\author{
Carlos Gómez Babillo, María Gómez Campillo \\ Universidad de Zaragoza \\ cgomez@unizar.es,mgc@unizar.es
}

Resumen: La globalización está produciendo un nuevo orden mundial que está afectando a las instituciones políticas, económicas, sociales, culturales y educativas. La universidad debe ser el motor de este cambio social y proponer un modelo educativo dinámico, flexible y estable que permita generar titulados preparados para afrontar los nuevos retos de la sociedad de la información y del conocimiento.

La universidad no solo debe proporcionar conocimientos básicos y especificos que preparen para el ejercicio de una profesión, sino que debe estimular el desarrollo de las capacidades de sus alumnos con competencias y habilidades sociales y técnicas que faciliten su posterior inserción laboral en un sistema productivo competitivo e innovador.

Palabras clave: globalización, mercado laboral, conocimiento, competencias, capacidades, habilidades.

Challenges and Changes in University Organization: Towards a New Model of Teaching and Learning

Abstract: Globalization is producing a new world order that is affecting political, economic, social, cultural and educational institutions. Universities need to be the engine of this social change and to provide a dynamic, flexible and stable educational model which can generate a skilled workforce ready to face the new challenges of the 
information and knowledge society.

The university must provide not only basic and specific knowledge that prepares students to embark upon a given profession but should develop students' social and technical skills to facilitate their subsequent employment in a competitive and innovative production system that demands a skilled workforce.

Keywords: globalization, labour market, knowledge, skills, abilities. 


\section{Planteamiento introductorio}

Estas páginas recogen unas reflexiones críticas sobre los cambios y los retos a los que debe enfrentarse la universidad española para responder a las demandas y las necesidades de la sociedad, a partir de la revisión de las principales aportaciones teóricas y propuestas alternativas que se han realizado sobre el tema. Se consideran las directrices y estrategias emanadas de las instituciones responsables de la educación superior en la Unión Europea y España y los retos que plantea el Espacio Europeo de Educación Superior para los gobiernos y para los universitarios.

\section{La globalización de la universidad}

La globalización está produciendo un nuevo orden mundial que ha supuesto la ruptura de los viejos sistemas productivos y ha modificado las condiciones de intercambio de bienes y servicios, así como de capitales; se han generado nuevas instituciones que regulan las relaciones económicas y políticas a escala planetaria (Gómez Bahillo, 2011). Este cambio no ha afectado exclusivamente al ámbito de lo económico y a las estructuras productivas, sino que también ha determinado, o lo está haciendo, un nuevo orden ideológico, político, social y cultural.

Las redes informáticas han incrementado la universalización de los intercambios políticos, tecnológicos, económicos, culturales, personales, etc. La aldea global (McLuhan y Powers, 1990) se ha convertido en realidad en el mundo globalizado en donde la información y el conocimiento están al alcance de un mayor número de personas.

La repercusión de estos cambios en la estructura ocupacional se traduce, en primer lugar, en un trasvase de activos hacia los servicios especializados, que han pasado a convertirse en el sector dinamizador de la economía; en segundo lugar, en el aumento del desempleo entre aquellos activos cuyos conocimientos y habilidades han quedado desfasados como resultado de los procesos de reconversión y adecuación tecnológica y que no disponen de una formación polivalente o no actualizan sus conocimientos de forma permanente, $y$, en tercer lugar, en las dificultades de consecución de empleo, a corto y medio plazo, por parte de colectivos de población joven.

El mercado laboral demanda un nuevo perfil de profesionales y trabajadores: se valoran muy positivamente no solamente sus conocimientos, sino también sus actitudes, competencias y habilidades, y aquellas cualidades personales que permiten y facilitan la adaptación a los cambios que se producen en el contexto económico, social y cultural, y en el entorno productivo y empresarial. Por ello, los contenidos formativos deben adecuarse a estos cambios sociales y a las nece- 
sidades del sistema económico y del mercado laboral. Es preciso establecer un modelo educativo y formativo dinámico, flexible y estable que permita generar una mano de obra cualificada y preparada para afrontar los nuevos retos de un sistema productivo global, según propone la Estrategia Europea para la consecución del pleno empleo.

Las empresas buscan personas competentes, que sean eficaces y eficientes en la resolución de las tareas y en el tratamiento de conflictos, que sean capaces de tomar decisiones y estén preparadas para hacer frente a las eventualidades de la práctica profesional. Personas con conocimientos, habilidades y destrezas suficientes para desarrollar con éxito las tareas encomendadas. En segundo lugar, se exigen competencias y actitudes sociales relacionadas con la resolución de problemas y la capacidad de trabajo, la ambición profesional, etc. junto con otras actitudes vinculadas al propio trabajo, como la capacidad de organización, liderazgo y trabajo en equipo, aspectos que pueden tener tanta importancia como el conocimiento profesional y técnico (Gómez Bahillo, 2012).

La principal misión de la universidad es la creación y transmisión de la cultura, la formación y preparación de los profesionales para la actividad que deberían desarrollar posteriormente y la contribución al desarrollo de la ciencia (Ortega y Gasset, 2007). La universidad del siglo xxi debe transformarse y reconvertirse a la «nueva realidad» proporcionando saberes contextualizados y aplicados a las necesidades de la sociedad actual, sin que ello suponga una mercantilización del conocimiento ni el establecimiento de alianzas condicionantes entre la universidad y los agentes económicos y empresariales, que le impidan mantener su espíritu crítico y la libertad de investigación. Por el contrario, la universidad debe contribuir al mantenimiento de un sistema social democrático estable, estableciendo alianzas con las organizaciones y los agentes sociales, políticos y económicos, que garanticen los derechos humanos y la consecución de niveles de bienestar y calidad de vida para todos los ciudadanos, lo que no está reñido con la productividad, la rentabilidad, la eficacia y la eficiencia.

La misión de la universidad en un contexto globalizado (Unesco, 1998: 2122) consiste principalmente en educar, formar $y$ realizar investigaciones que contribuyan al desarrollo sostenible y a mejorar la sociedad y, para ello, la universidad debe:

- Preparar graduados cualificados y responsables, con conocimientos teóricos y prácticos adaptados a las necesidades presentes y futuras de la sociedad y de los sectores productivos.

- Constituir un espacio abierto para la formación superior que propicie el aprendizaje permanente. 
+ Promover, generar y difundir conocimientos por medio de la investigación, proporcionar las competencias técnicas adecuadas para favorecer el desarrollo cultural, social y económico de la sociedad.

+ Contribuir a comprender, interpretar, preservar, reforzar, fomentar y difundir las culturas nacionales y regionales, internacionales e históricas, en un contexto de pluralismo y diversidad cultural.

+ Proteger y consolidar los valores de la sociedad civil democrática, proporcionando perspectivas críticas y objetivas.

- Participar en el desarrollo y la mejora de la educación mediante la formación del personal docente.

La universidad debe ser el motor del cambio social, en consonancia con las innovaciones que se producen en el contexto mundial y en su entorno más inmediato, y responder a los grandes desafíos de la globalización. Además, debe asumir su compromiso social contribuyendo a consolidar la sociedad civil y a fortalecer sus instituciones, a través de la educación. «Vivimos en una sociedad donde la innovación, la información y el conocimiento son rasgos fundamentales y donde los sistemas de comunicación la hacen abierta e intercomunicada, produciéndose una sociedad red, como afirma M. Castells, al estar todo conectado con todo [... ] La universidad [...] debe ser una institución que funcione, por tanto, con la misma lógica que la sociedad que es su producto, y al tiempo que es también un factor de transformación de la misma» (Monreal, 2001: 280-281). La sociedad de la información ha revolucionado las formas de acceso al conocimiento, la red se ha convertido en la fuente prioritaria de saber e internet ha pasado a ocupar el monopolio de conocimiento que durante siglos ha tenido la institución universitaria.

La universidad debe internacionalizarse porque el conocimiento es global y el mismo mercado de trabajo al que van acceder sus titulados rebasa las fronteras nacionales. Una estrategia de internacionalización está siendo el Espacio Europeo de Educación Superior y el proyecto del Espacio Iberoamericano de Educación Superior ${ }^{1}$, a través de los cuales es posible la convergencia de los distintos ámbitos de conocimiento y saberes, el establecimiento de metodologías de enseñanzas y programas cada vez más unificados y el reconocimiento de titulaciones y cualificaciones, independientemente del país en donde se hayan obtenido.

1 El Espacio Iberoamericano del Conocimiento (EIC) es una iniciativa que surge en el marco de las Cumbres Iberoamericanas de Jefes de Estado y de Gobierno, que se celebran anualmente desde el año 1991. En la Cumbre celebrada en Salamanca, en octubre de 2005, se acordó «avanzar en la creación de un Espacio Iberoamericano del Conocimiento, orientado a la necesaria transformación de la educación superior, y articulado en torno a la investigación, el desarrollo y la innovación, condición necesaria para incrementar la productividad brindando mejor calidad y accesibilidad a los bienes y servicios para nuestros pueblos así como la competitividad internacional en nuestra región», y se encomendó su proceso de constitución a la Secretaría General Iberoamericana (SEGIB), a la Organización de Estados Iberoamericanos para la Educación, la Ciencia y la Cultura (OEI) y al Consejo Universitario Iberoamericano (CUIB). 
Este proceso de globalización de las enseñanzas universitarias se ha visto impulsado, además de por las estrategias políticas establecidas por las instituciones internacionales, por:

- La internacionalización de los stakeholders, los grupos de interés relacionados con las enseñanzas universitarias (patrocinadores, empresarios empleadores, docentes y estudiantes).

- El impacto de la tecnología en el proceso de generación y distribución de conocimientos y saberes, en las metodologías docentes y en el conjunto del proceso de enseñanza-aprendizaje en donde el profesor deja de ser el transmisor de la información para convertirse en el orientador de la búsqueda y el tratamiento de la información. Los materiales educativos tienen un soporte digital y, en la mayoría de las ocasiones, están disponibles en las plataformas digitales a las que el estudiante puede acceder libremente.

- La transnacionalización del conocimiento que, al estar disponible en la red, es accesible para cualquier persona y para instituciones y organizaciones de diferentes partes del mundo.

Pero la internacionalización de la universidad debe incluir también medidas estructurales relacionadas con:

- Los sistemas de acreditación y reconocimiento de las titulaciones, que deberán tener una dimensión transnacional, a través de agencias internacionales de acreditación y evaluación de la calidad. Es preciso establecer un sistema que asuma los aspectos positivos del proceso de acreditación de las escuelas de negocios a nivel internacional: AMBA (Asociation of MBA), EQUIS (European Quality Improvement System) y AACSB (American Association of Collegiated Schools of Business).

- La información internacional de la oferta educativa, con transparencia pública de las evaluaciones existentes respecto a la cualificación de su profesorado, los programas curriculares y su adecuación a las necesidades sociales y demandas empresariales, los métodos pedagógicos, el nivel de exigencia al alumnado, los resultados académicos y la inserción laboral de sus egresados, etc.

- La organización universitaria, promoviendo las titulaciones de grado, máster y doctorado desde varias universidades con la participación de profesores y alumnos de varios países.

- La ampliación del concepto de campus universitario incluyendo a centros y titulaciones en diferentes ciudades y países. 
Una perspectiva internacional obliga a las universidades a aumentar sus niveles de especialización y competitividad mediante:

- Una oferta formativa especializada y competitiva relacionada con la calidad de sus titulaciones, la cualificación y el reconocimiento internacional de su profesorado, la investigación de sus departamentos, la inserción laboral de sus egresados, etc.

+ Una metodología didáctica innovadora con el empleo de recursos tecnológicos que permitan la sustitución de clases presenciales y el aprendizaje repetitivo de contenidos por un trabajo tutorizado en el que el estudiante es el protagonista de su proceso de enseñanza-aprendizaje (Miguel Díaz, 2006).

+ La oferta de una formación permanente dirigida a profesionales y personas adultas que tienen necesidad de reciclarse.

- El establecimiento de patrocinios y acuerdos con empresas multinacionales que permitan establecer clústeres en determinadas materias.

La Declaración de la Sorbona de $1998^{2}$ y la Declaración de Bolonia de $1999^{3}$ marcan un hito en la formación universitaria europea, no solo por el comienzo de un proceso de convergencia hacia un Espacio Europeo de Educación Superior, sino por los cambios que se introducen en el proceso de enseñanza-aprendizaje en el ámbito de las titulaciones universitarias, que afectan tanto a los conocimientos y al desarrollo de competencias para la empleabilidad, como a la forma como estas se consiguen. Las universidades deben proponer contenidos curriculares que capaciten a los estudiantes para vivir en la sociedad del conocimiento y para desarrollar las competencias necesarias para el ejercicio profesional, con programas formativos y metodologías innovadoras, acordes con las herramientas e instrumentos que proporciona la sociedad de la información.

El Consejo Europeo de Lisboa del 2000 estableció como un objetivo estratégico para la Unión Europea convertirse en la «economía basada en el conocimiento más competitiva y dinámica del mundo, capaz de crecer económicamente de manera sostenible con más y mejores empleos y con mayor cohesión social». Para ello, propuso no solamente «una transformación radical de la economía europea», sino también «un programa ambicioso de modernización del bienestar social y de los sistemas educativos ${ }^{4}$.

2 Declaración Conjunta para la armonización del diseño del Sistema de Educación Superior Europeo.

3 Declaración Conjunta de los ministros europeos de educación, reunidos en Bolonia el 19 de junio de 1999, sobre el Espacio Europeo de la Enseñanza Superior.

4 Conclusiones de la Presidencia, Consejo Europeo de Lisboa, 23 y 24 de marzo de 2000. 
La Comisión Europea, dentro del programa Educación y Formación 2010 establece una serie de medidas dirigidas a transformar el sistema educativo y formativo en los países miembros, y adecuarlo a los cambios que se producen en la sociedad y la economía del conocimiento en el entorno europeo y mundial. En relación con las enseñanzas universitarias, la Comisión ha emitido diversas comunicaciones que contienen directrices políticas para la consecución de los objetivos previstos. Entre estas, destacamos «El papel de las universidades en la Europa del conocimiento» ${ }^{6}$, en donde se plantea la función que la universidad debe tener en la sociedad y la economía del conocimiento, y «Europa y la investigación fundamental ${ }^{7}$, en donde se presenta el estado de la investigación fundamental en Europa, se destacan los retos a los que se enfrenta y se proponen sugerencias para mejorar los resultados europeos en el sector.

Este nuevo orden mundial ha afectado también a la universidad española que «no ha quedado al margen del proceso de globalización, y se ha visto impulsada de forma lenta pero irrevocable a una necesidad de compaginar la respuesta a las necesidades territoriales, en sintonía con las correspondientes políticas autonómicas, con una creciente competitividad a nivel internacional en la captación de recursos, talento y oportunidades, elementos estos esenciales para un mejor desarrollo de la sociedad de la que forma parte» (Ministerio de Educación, 2011: 2).

Es necesario reinventar la universidad española ante la pérdida de credibilidad que ha experimentado su capacidad formativa durante estas últimas décadas, especialmente entre los sectores empresariales, que consideran que la formación universitaria pública «ofrece un exceso de contenidos teóricos escasamente actualizados $y$, sobre todo, con una escandalosa falta de orientación práctica [...]», mientras que «la formación profesional y las universidades privadas acaban mostrando, para los empleadores, una mejor imagen que la universidad pública en lo que se refiere a la orientación práctica de los estudios y en la transmisión de valores de control y disciplina» (Alonso, Fernández Rodríguez y Nyssen, 2009: 111 y 129$)$.

La universidad no puede ser generadora de un saber descontextualizado ni, por otra parte, ser promotora de unos valores propios de la sociedad de mercado: pragmatismo, mercantilización de sus actividades, intereses y planteamientos. La universidad debe cuestionar y proponer alternativas a los principios economicistas de las relaciones productivas e intentar que la lógica del capital no prevalezca sobre los derechos humanos y el bienestar y la calidad de vida de los ciudadanos. Como se señala en la Ley Orgánica 4/2007, «la reforma pretende ser un paso

5 Consejo de Europa. Informe Educación y Formación 2010.

6 COM (2003) 58 final, de 5 de febrero.

7 COM (2004) 9 final, de 14 de enero. 
adelante en la organización del sistema universitario hacia una estructura más abierta y flexible, que sitúe a las universidades españolas en una mejor posición para la cooperación interna y la competencia internacional, a través de la creación, transmisión, desarrollo y crítica del conocimiento científico y tecnológico y de la transferencia de sus beneficios a la sociedad».

La universidad española debe responder a una serie de retos, que podemos concretar en:

- Gestionar la diversidad y, para ello, debe contextualizar el conocimiento y preparar profesionales con capacidad de adaptación a los cambios del entorno social y profesional.

- Promover el desarrollo de la capacidad creativa e innovadora de los estudiantes frente al aprendizaje memorístico y repetitivo.

- Capacitar profesionalmente a los estudiantes en las demandas ocupacionales que requiere el desarrollo económico y productivo.

- Promover una educación integral y emocional de la persona.

- Personalizar la educación a través de los nuevos recursos didácticos.

- Educar en valores y actitudes éticas: promover el humanismo frente a la lógica del capital.

- Dar una nueva dimensión a la investigación, orientándola a contribuir a la solución de problemas conceptuales, responder a las necesidades de la sociedad y transformar y adecuar la estructura productiva.

Los cambios universitarios deben afectar, por tanto, al currículo, las infraestructuras, la gestión administrativa, los recursos y las metodologías didácticas (utilización de las TIC) y al desarrollo competencial de profesores y alumnos. Sin embargo, principalmente, la universidad debe ser creadora de conocimiento básico y especializado, a través de la investigación y transferencia tecnológica: «el avance de las ciencias y su diversificación hacen imprescindible una mayor especialización y una formación más intensiva en la investigación y la transferencia de conocimientos [...]. Todos los grandes retos actuales van a necesitar nuevos conocimientos y profesionales altamente preparados, así como una necesidad de actualización continua de los contenidos y sistemas de aprendizaje» (Ministerio de Educación, 2011:3).

La educación universitaria española se encuentra ante un nuevo reto y, para ello, se han aprobado estrategias de transformación de la institución académica para adecuarla al nuevo entorno global y europeo. La Estrategia Universidad 2015 tiene como objetivo prioritario «lograr mejores universidades, con un sistema formativo y unas actividades de investigación y transferencia de conocimientos 
de calidad y competitivas en el panorama europeo e internacional» (Ministerio de Educación, 2011: 5). Y, para ello, se proponen cuatro ámbitos de actuación:

\section{Ámbitos de actuación en la organización universitaria}

\begin{tabular}{|c|c|}
\hline $\begin{array}{l}\text { Misiones } \\
\text { - Formación } \\
\text { - Adaptación de las enseñanzas al } \\
\quad \text { EEES } \\
\text { - Formación a lo largo de la vida } \\
\text { - Investigación } \\
\text { - Transferencia del conocimiento y } \\
\text { - } \quad \text { Recnología } \\
\text { Responsabilidad Social Universitaria }\end{array}$ & $\begin{array}{l}\text { Personas } \\
\text { - } \quad \text { Personal docente o investigador } \\
\text { - } \quad \text { Pstudiantes } \\
\quad \text { Personal de administración y } \\
\text { servicios }\end{array}$ \\
\hline $\begin{array}{ll}\text { Fortalecimiento de capacidades } \\
\text { - } & \text { Gobernanza } \\
\text { - } & \text { Financiación } \\
\text { - } & \text { Internacionalización } \\
\text { - } & \text { Evaluación (individual y colectiva) } \\
\text { - } & \text { Comunicación universitaria }\end{array}$ & $\begin{array}{l}\text { Entorno } \\
\text { - Campus de Excelencia } \\
\quad \text { Internacional }\end{array}$ \\
\hline
\end{tabular}

Fuente: MEC Estrategia Universidad 2015, pág. 9

\section{La transmisión de conocimiento y la capacidad formativa de las enseñanzas universitarias}

La globalización está produciendo un debilitamiento del conocimiento abstracto y, por el contrario, un fortalecimiento del conocimiento y el saber contextualizado. En la actualidad se produce un desfase entre la oferta educativa y las necesidades de la sociedad de la información y del conocimiento; por eso, la universidad debe adecuar sus titulaciones y crear, transmitir y generar nuevos conocimientos relacionados con nuevas necesidades.

Durante los últimos treinta años, en los países más desarrollados se ha producido más conocimiento que en los cinco milenios anteriores. Nos encontramos ante una verdadera revolución del conocimiento que tiene, y sin duda continuará teniendo, una gran repercusión sobre la organización económica y social. La información y el conocimiento se han convertido en el nuevo objeto de la ciencia, de forma que la economía y el bienestar social dependen del nivel alcanzado en el desarrollo y la expansión de las denominadas tecnologías de la información y la comunicación.

El conocimiento no es solo una fuente de poder, sino que es también la principal fuerza productiva de las naciones. Todos los cambios importantes en la 
economía y en la sociedad están, de una u otra manera, ligados a su desarrollo y expansión. Drucker (1993) considera que el conocimiento ha permitido el paso de una sociedad capitalista a la sociedad de la información, y ha originado una nueva forma de gestión y administración, «la revolución gerencial». El conocimiento se ha convertido, por tanto, en el factor de producción más importante, incluso por delante del capital y el trabajo, lo que ha generado una nueva dinámica socioeconómica, que afecta a la misma estructura social y laboral, y origina dos tipos de trabajadores: los trabajadores del conocimiento, aquellos que poseen una educación superior, ostentan el poder y dirigen los medios de producción, y los trabajadores de servicios, que ocupan un papel subordinado y con una escasa capacidad de decisión estratégica.

Históricamente, la universidad ha sido la cuna de la ciencia y del conocimiento, y de ella emanaba la formación en aquellos saberes que eran considerados como básicos para formar parte de la clase social dirigente y perpetuar, a través de ella, la estructura social. Por ello, existían trabas selectivas para acceder a los estudios universitarios y, una vez dentro, el estudiante debía superar multitud de pruebas en las que debía demostrar su capacidad para aprender y la adquisición y asimilación de los conocimientos básicos de las ciencias y las letras.

Pero muchos de estos conocimientos y saberes se producían y transmitían de forma descontextualizada, casi siempre con escasas referencias a las necesidades de la sociedad y alejados de las demandas del mercado laboral. Los investigadores fijaban los temas objeto de estudio, establecían metodologías, ritmos de trabajo, etc. ajenos, en ocasiones, a los cambios y transformaciones políticas, socioeconómicas, tecnológicas y culturales del entorno. Y esto se debía a que «tradicionalmente, los planes de estudios universitarios han tenido como referencia a los contenidos de las diferentes áreas de conocimiento organizadas en asignaturas; y han estado enfocados a un tipo de formación pensada para que los alumnos dominen esos contenidos», mientras que en la actualidad, y dentro del Espacio Europeo de Educación Superior, el «perfil es definido más por la intencionalidad formativa (objetivos) y el enfoque metodológico (actividades, organización de la clase, recursos, etc.) que por el contenido» (Yániz, 2005: 7).

\subsection{La función del profesor universitario}

La función del profesor consiste fundamentalmente en dirigir el proceso de aprendizaje, que puede realizarse sin necesidad de que los estudiantes asistan necesariamente durante toda la jornada a los centros educativos, e incluso las consultas y las evaluaciones pueden hacerse directamente a través de internet. Las tecnologías de la información y la posibilidad de acceso generalizado a la red 
están permitiendo que el aprendizaje en el aula sea sustituido por metodologías en las que el autoaprendizaje ocupe un papel importante y en las que la relación entre profesor y alumno se realice de manera virtual. La universidad tiene que preparar a las jóvenes generaciones para dominar y aplicar la principal materia prima, el conocimiento, y para ello tiene que disponer de los medios, programas y herramientas necesarias para su función. Como afirma Zabalza (2006: 48), es necesario transformar la docencia universitaria de manera que «el eje fundamental no sea la enseñanza sino el aprendizaje. Un proceso de formación que se justifica no tanto en la oferta que se hace (la enseñanza) cuanto en los resultados efectivos que cada estudiante alcanza (aprendizaje). Proceso de aprendizaje que, además, no se produce en abstracto o centrado en los contenidos propios de cada disciplina, sino que ha de hacerse tomando en consideración el proceso que sigue cada aprendiz para apropiarse del conocimiento y el contexto en que dicho proceso se produce».

Las nuevas formas de enseñanza (Benito y Cruz, 2006) deben ir orientadas a preparar y desarrollar la capacidad del alumno para buscar, procesar e interpretar la información que recibe. La clase magistral transmisora de conocimiento debe ser sustituida por el aprendizaje de herramientas e instrumentos que capaciten al alumno para investigar y encontrar la información que precisa, para que sepa discriminarla y seleccionar la que necesita y para que pueda analizar e interpretar los hechos y fenómenos objeto de estudio, así como proponer soluciones y realizar propuestas. Es un largo proceso, ya que «la lección magistral sigue siendo la práctica pedagógica dominante en los centros universitarios españoles, aunque cada vez se acompaña más de la realización de ejercicios, la resolución de problemas y la discusión de casos prácticos. Se considera conveniente reforzar las enseñanzas de tipo práctico, ya sea en la modalidad de prácticas vinculadas a asignaturas, ya en la modalidad de prácticas preprofesionales externas o estancias en centros de trabajo. La tutoría profesor-estudiante, orientada a complementar las clases y el propio trabajo de los alumnos, se considera otra práctica que es preciso potenciar». Es necesario «dar mayor protagonismo al estudiante en su formación, fomentar el trabajo colaborativo, organizar la enseñanza en función de las competencias que se deban adquirir, potenciar la adquisición de herramientas de aprendizaje autónomo y permanente, etcétera [... . El EEES se percibe como una gran oportunidad para generalizar experiencias de innovación en metodologías que se están realizando en muchas universidades» (Ministerio de Educación, 2006a: 7, 9 y 58).

La educación a distancia ha adquirido un nuevo significado que enfatiza el aprendizaje interactivo. En la actualidad, las universidades y los centros de for- 
mación empresarial están diseñando y ofreciendo estudios de posgrado y de especialización a través de la red. Los avances tecnológicos anuncian transformaciones educativas sin precedentes en los próximos años; las enseñanzas en línea experimentarán, sin duda, un crecimiento en los estudios universitarios y especialmente en los cursos de formación de posgrado.

El profesor universitario debería estar menos tiempo con los libros y más en internet e investigando y conociendo lo que se está haciendo en otras universidades. Es necesario pasar de la enseñanza basada en la transmisión detallada de conocimientos y opiniones de diferentes escuelas y autores y con la mirada al pasado, a la internacionalización del conocimiento abierto a las nuevas necesidades y demandas de la sociedad. El perfil ideal de profesor universitario (Galán, 2007) es aquel que posee conocimientos transversales y está motivado para compaginar formación y conocimientos con actitudes para investigar, buscar y aprender, y destaca por sus habilidades sociales y por la capacidad de convicción y entusiasmo.

El rol del profesor universitario en la sociedad del conocimiento consiste principalmente en:

+ Producir conocimiento.

- Fomentar en el estudiante inquietudes y habilidades de investigación y producción de conocimiento, y enseñarle a aplicar los saberes recibidos y aprendidos a la solución de los problemas y necesidades de su entorno y de su quehacer profesional.

- «Enseñar a aprender», es decir, ayudar a desarrollar en el estudiante no solo su capacidad memorística y la preparación para el ejercicio de una profesión u ocupación, sino también una inteligencia creativa, innovadora, crítica y abierta a la interpretación de los cambios que se producen en el entorno y a la búsqueda de soluciones y alternativas.

Para ello, el docente universitario deber estar capacitado y poseer conocimientos y destrezas para:

- Transmitir «diferentes formas de trabajar las nuevas tecnologías en su disciplina y área de conocimiento específica, adaptando los recursos disponibles a los objetivos y procesos de aprendizaje de cada conocimiento».

- Utilizar «distintos recursos, como laboratorios de medios audiovisuales, redes, biblioteca, sala multimedia, etc.».

- Organizar el aula, «de manera que se conozcan y se optimicen las posibilidades de los recursos y se ajusten éstos a las metodologías utilizadas». 
- Dominar «las técnicas y medios para la formación en cualquier espacio y tiempo, combinando la formación presencial con la formación a distancia».

- Seleccionar adecuadamente «los materiales, además de capacidad para rehacer y estructurar los materiales ya existentes de manera que se adapten a sus necesidades, incluso para elaborar nuevos materiales».

- «Incorporar las posibilidades de las nuevas tecnologías en la investigación» (García Ruiz, 2006:259).

\subsection{La formación competencial, objetivo de las enseñanzas universitarias}

Las competencias son «una combinación de conocimientos, habilidades (intelectuales, manuales, sociales, etc.), actitudes y valores que capacitarán a un titulado para afrontar con garantías la resolución de problemas o la intervención en un asunto en un contexto académico, profesional o social determinado» (Ministerio de Educación, 2006bः6). Para la Oficina Internacional de Trabajo, las competencias incluyen los conocimientos, las aptitudes profesionales y el saber hacer que una persona tiene y aplica en un contexto específico (OIT, 2005: 4).

En el proyecto Tuning ${ }^{8}$ se señala que las competencias son una combinación de conocimiento, comprensión, capacidades y habilidades. Las competencias «se entienden como conocer y comprender (conocimiento teórico de un campo académico, la capacidad de conocer y comprender), saber cómo actuar (la aplicación práctica y operativa del conocimiento a ciertas situaciones), saber cómo ser (los valores como parte integrante de la forma de percibir a los otros y vivir en un contexto social). Las competencias representan una combinación de atributos (con respecto al conocimiento y sus aplicaciones, aptitudes, destrezas y responsabilidades) que describen el nivel o grado de suficiencia con que una persona es capaz de desempeñarlos» (González y Wagenaar, 2003: 15).

Competencia es, por tanto, la capacidad para actuar sobre la realidad con eficiencia, eficacia y satisfacción. Cada competencia integra habilidades, aptitudes y conocimientos básicos, y se desarrolla a través de experiencias de aprendizaje en cuyo campo de conocimiento se integran diferentes tipos de saberes: conocimientos (SABER), procedimientos (SABER HACER) y actitudes (SER). Se trata de utilizar los conocimientos, habilidades y actitudes en contextos situacionales

8 Proyecto Tuning Educational Structures in Europe, coordinado por Julia González y Robert Wagenaar, de las Universidades de Deusto (España) y de Groningen (Países Bajos) respectivamente. Es un proyecto desarrollado por 100 universidades de países integrantes de la Unión Europea, que trata de comprender y rediseñar los curricula a partir de puntos de referencia comunes. El proyecto propone competencias genéricas y específicas en cada área temática de los graduados de primer y segundo ciclo. 
y aplicar correctamente los aprendizajes ya adquiridos al trabajo cotidiano o a las nuevas situaciones.

Cuando hablamos de competencias nos referimos, por tanto, a:

+ Conductas organizadas existentes en la estructura mental de cada persona, relativamente estables y susceptibles de ser aplicadas en los momentos en que resulten necesarias.

- Comportamientos que algunas personas dominan mejor que otras, y que las hacen ser más eficaces en una determinada situación.

- Experiencias y dominio real de la tarea.

- Habilidades de comportamiento que conducen a un desempeño excelente.

- Conocimientos, habilidades, actitudes e intereses que, puestos en acción, diferencian a unas personas de otras.

Las competencias no son simplemente habilidades ni aptitudes o rasgos personales - aunque estas desempeñan un papel importante cuando las tareas que se deben realizar son nuevas o complejas-, sino el conjunto de conocimientos y capacidades que una persona puede activar y aplicar en su ámbito laboral a la hora de desempeñar tareas o resolver problemas. Estas no se adquieren simplemente a través de un diploma o título académico, sino que para su formación se requiere la práctica y experiencia laboral, y ciertas aptitudes personales.

Las competencias profesionales se refieren al conjunto de cualificaciones individuales consideradas imprescindibles para el eficaz desempeño de una profesión o actividad ocupacional.

\section{Desarrollo competencial}

\begin{tabular}{|l|l|l|}
\hline \multicolumn{1}{|c|}{ Disposición } & \multicolumn{1}{c|}{ Requisitos } & \multicolumn{1}{c|}{$\begin{array}{c}\text { Factores de los que depende } \\
\text { su desarrollo }\end{array}$} \\
\hline Saber & $\begin{array}{l}\text { Conjunto de } \\
\text { conocimientos técnicos y } \\
\text { relacionales que afectan } \\
\text { al comportamiento. }\end{array}$ & $\begin{array}{l}\text { Disponibilidad para «aprender } \\
\text { a aprender»y «aprender a } \\
\text { pensar». }\end{array}$ \\
\hline Saber actuar & $\begin{array}{l}\text { Capacidad para } \\
\text { asumir los objetivos } \\
\text { y actividades de la } \\
\text { administración. } \\
\text { Habilidades técnicas, } \\
\text { sociales y cognitivas para } \\
\text { poner en práctica los } \\
\text { conocimientos. }\end{array}$ & $\begin{array}{l}\text { Formación y preparación } \\
\text { técnica. } \\
\text { Aplicación de conocimientos y } \\
\text { saberes a las tareas y funciones. }\end{array}$ \\
\hline
\end{tabular}




\begin{tabular}{|l|l|l|}
\hline \multicolumn{1}{|c|}{ Disposición } & \multicolumn{1}{|c|}{ Requisitos } & \multicolumn{1}{c|}{$\begin{array}{c}\text { Factores de los que depende } \\
\text { su desarrollo }\end{array}$} \\
\hline Saber estar & $\begin{array}{l}\text { Conjunto de } \\
\text { actitudes acordes } \\
\text { con las principales } \\
\text { características del } \\
\text { entorno organizacional } \\
\text { y/o social (cultura, } \\
\text { normas, etc.). }\end{array}$ & $\begin{array}{l}\text { Valores, creencias y actitudes } \\
\text { que favorecen o dificultan } \\
\text { determinados comportamientos } \\
\text { en un contexto dado. }\end{array}$ \\
\hline Querer actuar & $\begin{array}{l}\text { Motivación para el logro. } \\
\text { Condición subjetiva y } \\
\text { situacional. }\end{array}$ & $\begin{array}{l}\text { Identificación con la acción que } \\
\text { se debe desarrollar. } \\
\text { Imagen sobre el propio grado de } \\
\text { efectividad. }\end{array}$ \\
\hline Poder actuar & $\begin{array}{l}\text { Reconocimiento de la acción y } \\
\text { la confianza en sí mismo para } \\
\text { desarrollar una acción con éxito. }\end{array}$ \\
\hline $\begin{array}{l}\text { Contextuales. } \\
\text { Recursos e instrumentos } \\
\text { disponibles personales y } \\
\text { materiales. }\end{array}$ & $\begin{array}{l}\text { Posibilidad real de poder llevar } \\
\text { la tarea a término, en función } \\
\text { de la capacidad personal y de las } \\
\text { posibilidades situacionales del } \\
\text { entorno. }\end{array}$ \\
\hline
\end{tabular}

Fuente: elaboración propia

Se ha pasado de la necesidad de saberes parciales, segmentados y especializados, adquiridos en el sistema educativo formal, a saberes más ágiles y amplios, que deben ser puestos a prueba constantemente y que se acreditan a través de la actividad cotidiana. Se trata de un conjunto de saberes necesarios para resolver cuestiones concretas, y que son resultado de la combinación de conocimientos especializados y de experiencias que provienen fundamentalmente del trabajo realizado con anterioridad y de las capacidades y habilidades del alumno.

En la actualidad, los empresarios «demandan más competencias generales que específicas. La educación universitaria tiene un déficit de especial relevancia en las siguientes: habilidad para resolver problemas, resistencia a la presión laboral, gestión del tiempo y habilidades para utilizar las TIC; toma de responsabilidades y decisiones, planificación, coordinación y organización. Las cuatro primeras son necesarias para desarrollar el trabajo individual y las cuatro últimas están relacionadas con el desarrollo colectivo del trabajo» (Riesco, 2008: 22)

Por tanto, la educación universitaria debe proporcionar al estudiante:

+ Conocimiento del entorno político, socioeconómico, cultural y empresarial a nivel mundial, nacional y local. 
- Capacidad analítica e interpretativa de los hechos, acontecimientos, procesos, procedimientos, etc.

- Capacidad explicativa para establecer causas y correlaciones aplicando el conocimiento y los saberes aprendidos.

- Capacidad resolutiva que permita proponer respuestas y soluciones a las cuestiones planteadas.

Pero, para que esto sea posible, es necesario un cambio radical en el profesorado y el alumnado respecto a sus formas de pensar (otras actitudes, creencias, percepciones, habilidades...), maneras de sentir (mayor autoestima, mejor autoconcepto, ciertos valores...), formas de relacionarse (más cooperación, menos jerarquía, más trabajo en equipo...) y nuevas maneras de actuar (nuevas formas de enseñar y de aprender, nuevas maneras de organizarse y hacer planes, más calidad, creatividad y productividad...) (Martínez González, 2010).

La Unión Europea ${ }^{9}$ propone un sistema formativo que, además de transmitir conocimientos, ayude a desarrollar competencias y habilidades sociales y profesionales que faciliten la inserción laboral en un sistema productivo y empresarial competitivo e innovador, que demanda una mano de obra cada vez más cualificada y capacitada. Para ello, se establecen un conjunto de instrumentos para la construcción de un espacio europeo de cualificaciones profesionales y de aprendizaje permanente.

El Marco Europeo de Cualificaciones para el Aprendizaje Permanente $(\mathrm{EQF})^{10}$, común a la enseñanza universitaria y formación profesional, permite mejorar la información en relación con las competencias y cualificaciones, especialmente, permite a los empresarios valorar mejor el contenido y la relevancia de estas en el mercado de trabajo, y a los trabajadores, ejercer la movilidad y que sus cualificaciones sean reconocidas en todo el entorno europeo, independientemente del país en el que las hayan adquirido. Se trata de un instrumento basado en el aprendizaje y en sus resultados, independientemente de su duración, y que introduce como indicadores de evaluación las destrezas, las competencias y los conocimientos, a través de ocho niveles de referencia que sirven para medir lo que la persona sabe, comprende y es capaz de hacer, y que deberán tenerse en cuenta, a partir del 2012, en todas las nuevas cualificaciones expedidas por los centros de enseñanza postsecundaria de la Unión Europea.

9 Decisión n. ${ }^{\circ}$ 2241/2004/CE del Parlamento Europeo y del Consejo de 15 de diciembre de 2004. El Consejo Europeo, sesiones de 16 y 17 de junio de 2005. Conclusiones de la Presidencia, pág. 2. Se planteó la necesidad de recuperar la Estrategia de Lisboa y se fijaron las directrices para el crecimiento y el empleo, entre las que se incluye la adaptación de la educación y formación a las nuevas necesidades laborales en materia de competencias.

10 Recomendación del Parlamento Europeo y del Consejo, de 23 de abril de 2008. 
En España, el proceso de adecuación de las enseñanzas universitarias a las directrices del Espacio Europeo de Educación Superior introdujo modificaciones importantes en el diseño de las titulaciones y de los currículos (Ministerio de Educación, 2003). El Real Decreto 55/2005, de 21 de enero, señala en su parte expositiva que «las enseñanzas oficiales del ciclo de Grado se regulan con un objetivo formativo claro, que no es otro que el de propiciar la consecución por los estudiantes de una formación universitaria que aúne conocimientos generales básicos y conocimientos transversales relacionados con su formación integral, junto con los conocimientos y capacidades específicos orientados a su incorporación al ámbito laboral». El Real Decreto 1393/2007 indica, en su parte expositiva, que los planes de estudios deberán contemplar las competencias que los estudiantes deberán haber adquirido al terminar la titulación, y establece las directrices para la elaboración de las memorias para la verificación de las enseñanzas universitarias oficiales, en las que se recogen las competencias generales y específicas que deberán adquirir los estudiantes para ser evaluados positivamente.

\section{Competencias básicas mínimas que deben adquirirse en las titulaciones universitarias}

\begin{tabular}{|l|l|}
\hline \multicolumn{1}{|c|}{ Grado } \\
\hline$+\quad$ Que los estudiantes hayan demostrado poseer y comprender conocimientos en \\
un área de estudio que parte de la base de la educación secundaria general, y \\
se suele encontrar a un nivel que, si bien se apoya en libros de texto avanzados, \\
incluye también algunos aspectos que implican conocimientos procedentes de la \\
vanguardia de su campo de estudio. \\
Que los estudiantes sepan aplicar sus conocimientos a su trabajo o vocación de \\
una forma profesional y posean las competencias que suelen demostrarse por \\
medio de la elaboración y defensa de argumentos y la resolución de problemas \\
dentro de su área de estudio. \\
Que los estudiantes tengan la capacidad de reunir e interpretar datos relevantes \\
(normalmente dentro de su área de estudio) para emitir juicios que incluyan una \\
reflexión sobre temas relevantes de índole social, científica o ética. \\
Que los estudiantes puedan transmitir información, ideas, problemas y solu- \\
ciones a un público tanto especializado como no especializado. \\
Que los estudiantes hayan desarrollado aquellas habilidades de aprendizaje nec- \\
esarias para emprender estudios posteriores con un alto grado de autonomía. \\
\hline \multicolumn{1}{c}{ Máster } \\
\hline $\begin{array}{l}\text { Que los estudiantes sepan aplicar los conocimientos adquiridos y su capacidad } \\
\text { de resolución de problemas en entornos nuevos o poco conocidos dentro de } \\
\text { contextos más amplios (o multidisciplinares) relacionados con su área de estudio. }\end{array}$ \\
\hline
\end{tabular}


Máster

- Que los estudiantes sean capaces de integrar conocimientos y enfrentarse a la complejidad de formular juicios a partir de una información que, siendo incompleta o limitada, incluya reflexiones sobre las responsabilidades sociales y éticas vinculadas a la aplicación de sus conocimientos y juicios.

- Que los estudiantes sepan comunicar sus conclusiones - y los conocimientos y razones últimas que las sustentan- a públicos especializados y no especializados de un modo claro y sin ambigüedades.

- Que los estudiantes posean las habilidades de aprendizaje que les permitan continuar estudiando de un modo que habrá de ser en gran medida autodirigido o autónomo.

\section{Doctorado}

- Que los estudiantes hayan demostrado una comprensión sistemática de un campo de estudio y el dominio de las habilidades y métodos de investigación relacionados con dicho campo.

- Que los estudiantes hayan demostrado la capacidad de concebir, diseñar, poner en práctica y adoptar un proceso sustancial de investigación con seriedad académica.

- Que los estudiantes hayan realizado una contribución a través de una investigación original que amplíe las fronteras del conocimiento desarrollando un corpus sustancial, del que parte merezca la publicación referenciada a nivel nacional o internacional.

+ Que los estudiantes sean capaces de realizar un análisis crítico, evaluación y síntesis de ideas nuevas y complejas.

- Que los estudiantes sepan comunicarse con sus colegas, con la comunidad académica en su conjunto y con la sociedad en general acerca de sus áreas de conocimiento.

- Que se les suponga capaces de fomentar, en contextos académicos y profesionales, el avance tecnológico, social o cultural dentro de una sociedad basada en el conocimiento.

Fuente: Real Decreto 1393/2007, de 29 de octubre. Anexo I. Punto 3

Rodríguez Esteban y Vieira Aller (2009: 27-47), tomando la tipología que establece el proyecto Tuning, establecen una serie de competencias genéricas y específicas para analizar la formación que han recibido los graduados en la Universidad de León, que pueden hacerse extensivas al conjunto de las enseñanzas universitarias españolas y cuyo modelo presentamos a continuación. 


\section{Competencias que deben obtener los graduados universitarios al finalizar sus estudios}

\begin{tabular}{|c|c|}
\hline \multicolumn{2}{|c|}{ Competencias genéricas } \\
\hline $\begin{array}{l}\text { Competencias instrumentales } \\
\text { Capacidades cognitivas, metodológi- } \\
\text { cas, tecnológicas y lingüísticas }\end{array}$ & $\begin{array}{l}\text { + Capacidad reflexiva sobre su propio tra- } \\
\text { bajo. } \\
\text { + Cultura general. } \\
\text { + } \\
\text { Idiomas: conocimiento de idiomas extran- } \\
\text { jeros. } \\
\text { Toma de decisiones y solución de proble- } \\
\text { mas: localización del problema, identificar } \\
\text { causas y alternativas de solución, selección } \\
\text { y evaluación de la más idónea. } \\
\text { Expresión oral: saber expresarse con clari- } \\
\text { dad en conversaciones o debates adecu- } \\
\text { ando el estilo del lenguaje al interlocutor } \\
\text { y utilizando vocabulario específico y rel- } \\
\text { evante. } \\
\text { Expresión escrita: saber expresarse con } \\
\text { claridad en la redacción de escritos adecu- } \\
\text { ando el estilo del lenguaje al interlocutor } \\
\text { y utilizando vocabulario específico y rel- } \\
\text { evante. } \\
\text { Informática: buen conocimiento de las } \\
\text { herramientas más comunes de informáti- } \\
\text { ca. } \\
\text { Habilidad de documentación: consulta de } \\
\text { bases de datos relevantes en el campo pro- } \\
\text { fesional, consulta de revistas específicas, } \\
\text { navegación experta por internet... }\end{array}$ \\
\hline $\begin{array}{l}\text { Competencias interpersonales } \\
\text { Capacidades individuales y habili- } \\
\text { dades sociales (interacción social y co- } \\
\text { operación) }\end{array}$ & $\begin{array}{l}\text { + Trabajo en equipo: capacidad de compro- } \\
\text { miso con un equipo, hábito de colabo- } \\
\text { ración y trabajo solucionando los conflic- } \\
\text { tos que puedan surgir. } \\
\text { + Razonamiento cotidiano: capacidad de } \\
\text { buscar argumentos de sentido contrario a } \\
\text { la propia opinión. } \\
\text { Pensamiento crítico: capacidad de anal- } \\
\text { izar, sintetizar y extraer conclusiones de } \\
\text { un artículo. }\end{array}$ \\
\hline
\end{tabular}




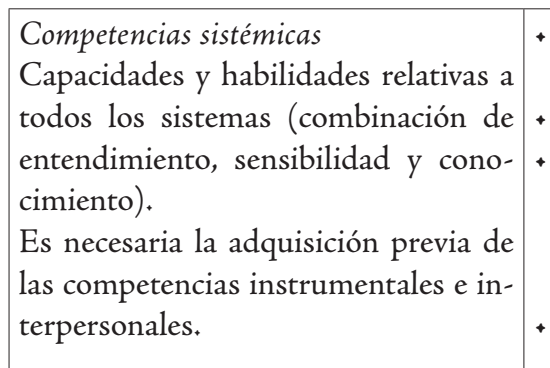
tividades, identificar las críticas, establecer plazos y cumplirlos.

Creatividad: capacidad de innovación, iniciativa, fomento de ideas e inventiva.

Competencias especificas

- Formación práctica in situ (prácticum, estancias en empresas, etc.).

- Formación práctica obligatoria: curricular (créditos prácticos).

+ Formación teórica: optativas.

+ Formación teórica: obligatorias.

Fuente: Rodríguez Esteban y Vieira Aller (2009: 27-47)

Las competencias genéricas son aquellas que deben adquirir todos los estudiantes universitarios, independientemente de la titulación que estén cursando, y que les capacitan para desenvolverse sin dificultad en la sociedad del conocimiento, mientras que las específicas son aquellas que hacen referencia a los conocimientos y destrezas para el desempeño de una determinada profesión o actividad. La institución universitaria debe apostar por que los conocimientos y capacidades que el alumno va adquiriendo en las aulas tengan una aplicación práctica y, para ello, el prácticum y las prácticas externas deben ocupar una parte importante de los currículos de todas las titulaciones. Los sistemas de evaluación deben centrarse no solo en los conocimientos que el alumno haya adquirido, sino muy especialmente en las competencias que haya desarrollado (Poyatos, 2009).

\section{Conclusión}

La universidad del siglo xxi debe proporcionar saberes contextualizados y aplicados a las necesidades de la sociedad actual y ser motor del cambio social en consonancia con las innovaciones que se producen en el contexto mundial y los grandes desafíos de la globalización.

La universidad debe internacionalizarse. Los sistemas de acreditación y reconocimiento de las titulaciones deberán tener una dimensión transnacional, a través de agencias internacionales de acreditación y evaluación de la calidad, y deberá evaluarse la oferta formativa especializada y competitiva por la calidad de las titulaciones, la cualificación y el reconocimiento internacional del profesora- 
do, la investigación de los departamentos, la inserción laboral de los egresados, entre otros.

Es necesario pasar de la enseñanza basada en la transmisión detallada de conocimientos y opiniones de diferentes escuelas y autores y con la mirada al pasado, a la internacionalización del conocimiento abierto a las nuevas necesidades y demandas de la sociedad. El profesor debe ser el orientador de la búsqueda y el tratamiento de la información con una metodología didáctica innovadora y recursos tecnológicos que sustituyan las clases presenciales y el aprendizaje repetitivo de contenidos por un trabajo tutorizado, en el que el estudiante sea el protagonista de su proceso de enseñanza-aprendizaje.

Los cambios universitarios deben afectar, por tanto, al currículo, las infraestructuras, la gestión administrativa, los recursos y las metodologías didácticas (utilización de las TIC) y el desarrollo competencial de profesores y alumnos. Los contenidos curriculares deben capacitar a los estudiantes para vivir en la sociedad del conocimiento y para desarrollar las competencias necesarias para el ejercicio profesional.

Los titulados universitarios deben tener conocimiento del entorno político, económico, social, cultural y empresarial; capacidad analítica e interpretativa de la realidad; capacidad explicativa de las causas y correlaciones entre los diferentes hechos, fenómenos y acontecimientos, y capacidad resolutiva para proponer soluciones y alternativas.

Las directrices europeas dentro del Espacio Europeo de Educación Superior van esa dirección. Es un reto que la universidad debe asumir y, para ello, precisa recursos humanos y tecnológicos suficientes para afrontar este gran cambio y la actitud favorable y la disponibilidad de profesores y alumnos.

\section{Bibliografía}

Alonso, Luis Enrique; Fernández Rodríguez, Carlos y Nyssen, José María (2009). El debate de las competencias. Una investigación cualitativa en torno a la educación superior y el mercado de trabajo en España. Madrid: Aneca.

Benito CApa, Águeda y Cruz, Ana (coords.) (2005). Nuevas claves para la Docencia Universitaria en el Espacio Europeo de Educación Superior. Madrid: Narcea Ediciones.

Drucker, Peter (1993). La sociedad postcapitalista. Barcelona: Apóstrofe. Galán, A. (2007). El perfil del profesor universitario. Situación actual y retos de futuro. Madrid: Ediciones Encuentro. 
García Ruiz, María Rosa (2006). «Las competencias de los alumnos universitarios». Revista Interuniversitaria de Formación del Profesorado, 20(3), 253269.

Gómez Bahillo, Carlos (2011). «La globalización y el nuevo orden/desorden mundial. La crisis de 2008». Sociedad y Utopía, Revista de Ciencias Sociales, $37,115-138$.

- (2012). «La formación profesional en el sistema productivo» en Antonio TRINidAd Requena y Javier Gómez González (coords.) (2012). Sociedad, familia y educación. Una introducción a la Sociología de la Educación. Madrid: Tecnos.

González, Julia y Wagenaar, Robert (ed.) (2003). Tuning Educational Structures in Europe. Informe Final. Fase Uno. Bilbao: Universidad de Deusto.

Martínez González, José Alberto (2010). «La naturaleza de las competencias en el espacio europeo de educación superior». Cuadernos de Educación y Desarrollo, vol. 2 n. 22 .

McLuhan, Marshall y Powers, Bruce R. (1990). La aldea global: transformaciones en la vida y los medios de comunicación mundiales en el siglo XXI. Barcelona: Editorial Gedisa.

Miguel Díaz, Mario de (coord.) (2006). Metodologías de enseñanza y aprendizaje para el desarrollo de competencias. Orientaciones para el profesorado universitario ante el Espacio Europeo de Educación Superior. Madrid: Alianza Editorial.

Monreal, Juan (2001). «Reflexiones sobre el Informe Universidad 2000». REIS, 93, 277-286.

OIT (2005). Recomendación sobre el desarrollo de los recursos bumanos: educación, formación y aprendizaje permanente. Ginebra: OIT.

Ortega y Gasset, José (2007). Misión de la universidad. Madrid: Biblioteca Nueva.

Poyatos Lorente, Manuel et alii (2009). Evolución del sistema educativo espanol: de la educación centralista a la educación por competencias. Granada: Editorial Adhara.

Riesco González, Manuel (2008). «El enfoque por competencias en el EEES y sus implicaciones en la enseñanza y el aprendizaje». Tendencias Pedagógicas, 13, 79-105.

Rodríguez Esteban, Agustín y Vieira Aller, María José (2009). «La formación en competencias en la universidad: un estudio empírico sobre su tipología». Revista de Investigación Educativa, vol. 27, 1, 27-47.

Yaniz Álvarez de Eulate, Concepción (2006). La función docente del profesorado en el marco del EEES. Zaragoza: Instituto de Ciencias de la Educación. Documentos $n+^{\circ} 3$. 
Zabalza Beraza, Miguel Ángel (2006). «La convergencia como oportunidad para mejorar la docencia universitaria». Revista Interuniversitaria de Formación del Profesorado, 20 (3), 37-69.

\section{Fuentes documentales}

Comunicación de la Comisión: «El papel de las universidades en la Europa del conocimiento» (COM (2003) 58 final de 5 de febrero). <http://europa. eu.int/eur-lex/es/com/cnc/2003/com2003_0058es01.pdf $>$.

Comunicación de la Comisiónः «Europa y la investigación fundamental» (COM (2004) 9 final de 14 de enero). <http://europa.eu.int/comm/research/ press/2004/pdf/ acte_es_version_final_15janv_04.pdf $>$.

Conclusiones de la Presidencia, Consejo Europeo de Lisboa, 23 y 24 de marzo de 2000. <http://www.europarl.europa.eu/summits/lis1_es.htm>.

Decisión n+* 2241/2004/CE del Parlamento Europeo y del Consejo de 15 de diciembre de 2004, relativa a la creación de un Marco Comunitario Único para la Transparencia de las Cualificaciones y Competencias. http://eur-lex.europa.eu/LexUriServ/LexUriServ.do?uri=OJःL:2004:390:0006:0020:EsะPDF

Conclusiones de la Presidencia, Consejo Europeo de Bruselas, 16 y 17 de junio de 2005. <http://ue.eu.int/ueDocs/cms_Data/docs/pressData/es/ ec/85347.pdf>.

Declaración Conjunta para la armonización del diseño del Sistema de Educación Superior Europeo (a cargo de los cuatros ministros representantes de Francia, Alemania, Italia y el Reino Unido). La Sorbona, París, 25 de mayo de 1998. <https://ujiapps.uji.es/estudis/eees/titols/documents/docs/sorbona.pdf>.

Declaración Conjunta de los ministros europeos de educación, reunidos en Bolonia el 19 de junio de 1999, sobre el Espacio Europeo de la Enseñanza Superior. <http://www.eees.es/pdf/Bolonia_ES.pdf>.

Informe Educación y Formación 2010 del Consejo de la Comisión Europea. Publicado en Diario Oficial de las Comunidades Europeas, n. ${ }^{\circ}$ 104, 30/04/2004, pp. 1-19.

Ministerio de Educación y Cultura (2003). La Integración del Sistema Universitario Español en el Espacio Europeo de Enseñanza Superior. Documento Marco. <http://www.uhu.es/convergencia_europea/documentos/documentos-2007/docmarco_MEC_feb2003.pdf>.

Ministerio de Educación (2006a). Propuestas para la renovación de las Metodologías Educativas en la Universidad. Madrid. Consejo de Coordinación Universitaria (Comisión para la Renovación de las Metodologías Educativas en la Universidad). <http://www.uemc,es/es/UEMC/Organizacion/Vicerrec- 
toradoOrdenacionAcademicaCalidadEmpleo/EEES/Documents/Propuesta\%20de\%20renovacion\%20de\%20las\%20metodologias\%20docentes.pdf $>$.

Ministerio de Educación (2006b). Directrices para la elaboración de títulos universitarios de grado y máster. Documento de Trabajo. 21 de diciembre de 2006. <http://firgoa.usc.es/drupal/files/directrices.pdf $>$.

Ministerio de Educación. Ley Orgánica 4/2007, de 12 de abril, por la que se modifica la Ley Orgánica 6/2001, de 21 de diciembre, de Universidades (BOE del 13 de abril).

Ministerio de Educación (2011). Estrategia Universidad 2015. Resumen. Madrid. <http://trabajadoresucm.files.wordpress.com/2011/12/estrategiauniversidad2015-resumen.pdf $>$.

Proyecto Tuning Educational Structures in Europe, coordinado por Julia González y Robert Wagenaar, de las Universidades de Deusto (España) y de Groningen (Países Bajos) respectivamente. <http://tuning.unideusto.org/tuningeu, $y$ www.rug.nl/let/tuningeu $>$.

Real Decreto 55/2005, de 21 de enero, por el que se establecen las estructuras de enseñanzas y se regulan los estudios universitarios de grado, de acuerdo con las líneas generales emanadas del EEES (BOE de 25 de enero).

Real Decreto 1393/2007, de 29 de octubre, por el que se regula la ordenación de las Enseñanzas Universitarias Oficiales en España (BOE 30 de octubre).

Recomendación del Parlamento Europeo y del Consejo, de 23 de abril de 2008, relativa a la creación del Marco Europeo de Cualificaciones para el aprendizaje permanente (EQF). (Diario Oficial C 111 de 6.5.2008). <http://ec,europa. eu/education/pub/pdf/general/eqf/broch_es.pdf $>$.

Unesco (1998). Conferencia Mundial sobre la Educación Superior. La educación superior en el siglo XXI. Visión y acción. París, 9 de octubre de 1998. <http:// www.unesco.org/education/educprog/wche/declaration_spa.htm $>$. 